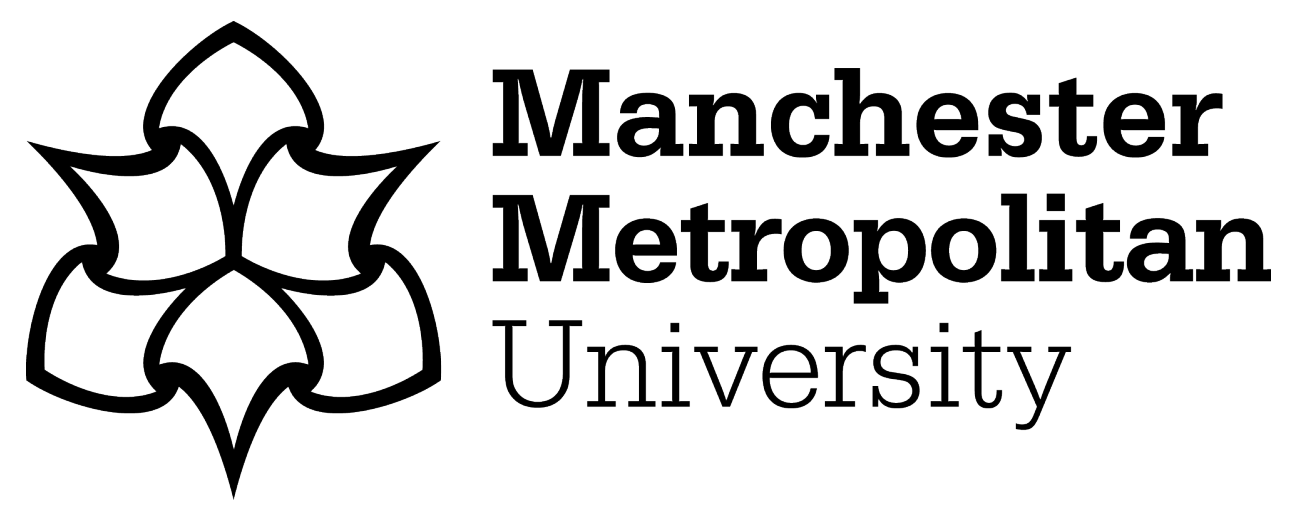

Foley, M and Cummins, I (2018) Reporting sexual violence on mental health wards. Journal of Adult Protection, 20 (2). pp. 93-100. ISSN 1466-8203

Downloaded from: https://e-space.mmu.ac.uk/620704/

Publisher: Emerald

DOI: https://doi.org/10.1108/JAP-11-2017-0036

Please cite the published version 


\section{Reporting Sexual Violence on Mental Health Wards.}

\section{Abstract \\ Purpose}

This paper reports the findings of a scoping study that explored the extent of recorded sexual violence perpetrated on inpatients on mental health units.

\section{Design/methodology/approach}

A Freedom of Information Act (FOI) request was sent to 45 Police forces. The FOI asked for the number of recorded offences of rape and sexual assault by penetration for the five years 2010- 2015. Following the responses from the Police, a similar FOI request was sent to Mental Health (MH) Trusts.

\section{Findings}

There were significant variations in the way that both Police forces and $(\mathrm{MH})$ Trusts recorded this information.

\section{Research limitations/implications}

The research highlights variation and inadequacy of current recording practices in relation to sexual offences committed against inpatients on mental health units.

\section{Practical implications}

There needs to be more consistent system of recording of allegations of sexual assault and responses to them by agencies. In the Trust recording of these incidents, it is recommended that a specific category of sexual violence is created. On a national level, the Office for National Statistics (ONS) should produce a national data set that records the number of rapes that are committed in mental health inpatient units.

\section{Originality/value}

The paper highlights the 'gap' of information in relation to recorded rape and may indicate that complainants with a history of mental illness are less likely to have their allegation recorded as a crime 




\section{Introduction}

This paper examines the reporting of incidents of rape and sexual assault on mental health inpatient units. Stenhouse (2013) uses the term "sanctuary harm"to capture the potentially damaging impact of abuse or violence that occurs to patients who have been admitted to a mental health unit. Jones et al (2010) see wards as both safe and unsafe places. It should be noted that people with mental health problems are much more likely to be victims of crime and abuse than the general population. Therefore, the mental health unit might be a safer place, particularly if individuals have good relationships with staff and are familiar with the environment and other patients. Jones et al's work (2010) indicates that factors such as unfamiliarity with the staff group and witnessing the use of restraint and seclusion were factors that increased anxiety amongst patients. $61 \%$ of female patients reported harassment or abuse during an admission to a mixed sex psychiatric ward (Clarke, 2008).

\section{Sexual violence and mental health}

People who are using mental health services are more likely than other groups in the population to have experienced sexual violence. It is, therefore, vitally important that mental health services develop systems that respond to these issues. This would include not only ensuring that all mental health staff are aware of the potential impact of sexual violence. In addition, staff need to have an awareness of the potential links between experience of sexual violence and the development of mental health problems. In their examination of the experiences of physical and sexual violence Scott et al (2015) identified six groups within the population:

- Relatively little experience of violence or abuse - this is the majority of the population, roughly $75 \%$. However, within this group $14 \%$ reported having been bullied at some point.

- Physical violence from a partner - this group made up $10 \%$ of the population. Over $60 \%$ of this group reported that they had been kicked, bit or hit by a partner.

- Extensive physical violence from a partner - this group made up $2 \%$ of the population. Over $80 \%$ of this group had been threatened with death.

- Sexual violence as a child (not in adulthood) - this group made up 5\% of the population. $66 \%$ had been "touched sexually as a child" 
- Sexual violence as an adult (and sometimes also in childhood) - this group made up 3\% of the population. 30\% had "non-consensual intercourse in adulthood"

- Extensive physical and sexual violence as an adult and child - this group made up 4\% of the population. As well as being subjected to physical violence such as being hit, bitten and kicked by a partner, individuals in this group had suffered "very high levels of severe sexual violence"

(adapted from Scott et al, 2015 page 5).

People who have been subjected to physical and sexual violence are more likely than other groups to develop mental health problems. These difficulties include common mental disorders, eating disorders, psychosis and Post Traumatic Stress Disorder (PTSD) (Scott et al, 2015). For example, Scott et al (2015) note that people who have been violently assaulted or abused are fifteen times more likely to make an attempt to take their own life than the general population. Rates of self-harm are five times higher amongst this group. $84 \%$ of those in the group who had been subject to "extensive physical and sexual violence" were women. $12 \%$ of this group had been admitted to a specialist mental health unit at some point. This review concludes that the long term impact of violence in all its forms should be viewed as public health issue. The impact of violence can be seen across both physical and mental health. There are well established links with other concerns including, alcohol and drug dependency, smoking, obesity and childhood development.

People with mental health problems are at greater risk of victimisation than the general population (Choe et al 2008). Bengsten-Tops and Ehliasson (2011) carried out a study involving 174 patients. $67 \%$ of this cohort had been the victims in adulthood. $39 \%$ had been threatened at some point. $51 \%$ had been assaulted and $32 \%$ had been the victims of sexual assault. $33 \%$ of the cohort had been a victim in the previous twelve months $-15 \%$ had been subjected to sexual violence in that period. Women reported greater exposure to violence than men. Khalifeh et al (2015) note the focus of research on violence and serious mental illness has been on debates around whether people with mental health problems are more likely than other groups to commit such offences. This is clearly a very important area but it does divert attention from the experiences of people with mental health problems as victims of serious violent crime. The authors note that the relationship between serious mental illness and experiencing violence is a complex one that is most likely to be bi-direction. Service-user histories of 
trauma are often not acknowledged by professionals (Howard et al, 2010) or where they are there is not an appropriate response (Nyamie et al, 2013). Khalifeh et al (2015) conclude that men and women who experience severe mental illness and are in contact with psychiatric services are 2-8 times more likely to experience domestic violence or sexual assault. This study also concluded that these experiences of violence could be a potential trigger for suicidal ideation. In the report At Risk yet Dismissed (Pettitt et al, 2013) 9\% of victims reported crimes that had taken place in psychiatric settings. This study is based on a series of interviews with people with mental health problems who had been victims of crime. These interviews reveal the impact of violence with participants reporting that they felt fear, shame and embarrassment in the aftermath. The negative impact on mental health was also documented with participants reporting feelings of distress. In some cases, the mental health of victims deteriorated and the crime triggered a relapse and subsequent admission to hospital. The issue of reporting was also examined in this study. Participants reported that one of the factors in their decision not to report was a fear that they would not be believed. Those who decided to report the assault to the police indicated that support from friends, family and professionals was a factor alongside a previous positive experience with the police. In addition, the concern that the offences might escalate and the wish to protect others were all positive factors in this process. This study also highlighted the concerns that individuals had that would prevent the reporting incidents to the police. A previous negative experience with the police was a factor here. However, the overarching concern was that they would not be believed. The interviewees were extremely conscious that the fact that they had a mental health problem might potentially be used as a basis for discrediting their account. It is highly regrettable that many thought that they might even be detained under the Mental Health Act (MHA) 1983 if they reported a crime.

\section{Patient Safety}

The potential impact of sexual violence is such that it must be a priority issue for mental health services. There are two elements to this. The first is that those using mental health services may have been subjected to sexual violence. The second is that in patients may be at greater risk. All patients are owed a duty of care, this extends to ensuring personal safety whilst an inpatient. A Care Quality Commission (CQC) report in 2014 raised concerns over the widespread use of petty rules in psychiatric units, for example, banning the use of mobile phones and the internet. These have become key features of modern life and it couldbe argued - citizenship. They are also important ways of maintaining contact with friends and families. 
Goffman (1968) in his outline of the culture of the "total institution" highlighted the social and physical isolation that they created. The CQC report noted that in $13 \%$ of cases no one actually knew why the rule had been introduced in the first place. There have been ongoing concerns about the physical environment of mental health wards. Quirk and Lelliot (2001) found that patients were concerned about life on the wards - they reported concerns about the lack of meaningful activity. Alongside this, wards were described as dirty and the food was poor. In addition, patients reported that they were concerned by the availability and use of street drugs on the wards. Patients in this study reported that they were concerned for their physical safety. This is a hugely important issue. Risk to self - in one form or another- is the core reason for admission to hospital. The personal safety of patients has to be a priority issue for all those working in mental health care. In the inpatient setting, if patients are concerned that they are not physically safety then this will almost certainly have an impact on their mental health. In the context of this research, these factors can contribute to an environment where individuals feel more vulnerable.

Sweeney et al (2016) note in their review of Trauma Informed Approaches (TIA) that mental health systems can be sites of initial trauma or further trauma. Trauma can be a single event or a series of events. The definition is broad enough to include experiences of interpersonal violence, for example sexual abuse and physical assault as well social trauma such as inequality and marginalisation. Individuals may experience personal trauma whilst at the same time being caught up in community traumas. For example, individuals fleeing a war zone may be subjected to forms of interpersonal violence alongside the trauma of being a member of a persecuted group in society. These traumas can be compounded by the reaction of authorities or public services to the individuals and family. Retraumatisation occurs when a person experiences an event that triggers memories of a past traumatic event. The current event then triggers similarly emotional responses - fear, anxiety, depersonalisation - to those of the original event. Bloom and Farragher, 2010 argue that current mental health systems with their inbuilt focus on coercion and control have the potential to retraumatise survivors. This may occur via the use of physical restraint and seclusion. There are pressures - for example to cooperate with the terms of a Community Treatment Order (CTO) or to take medication (Sweeney et al 2016). 
Bloom and Farragher, (2010) argue that staff are required to work in "trauma- organised systems". Social workers and mental health professional enter these systems with the aim of relieving suffering rather than inflicting it. The long term impact on staff is the development of therapeutic pessimism or a loss of a sense of why they entered the profession in the first place. This is superbly illustrated in Filer's (2013) novel The Shock of the Fall where the staff are unable to communicate with a young patient Matthew so do not understand the extent of his mental distress. Paterson (2014) argues TIA is a systems approach that focuses on how the impact of trauma affects has impact across the fields of neurological, biological, psychological and social development. Services need to organised and delivered in ways that recognise that service users have potentially been exposed to various forms of trauma. The services need to thus ensure that any they delivered ethically informed and safe services. TIA mental health services are thus strengths based approaches. They seek to recognise that complex behaviours have a function in both helping survivors manage and respond to situational triggers. Spandler's (1996) work on self -harm is an example of this approach. It puts forward an approach that rather than seeing self-harm as a behaviour that needs to be managed, views it as a behaviour that has intrinsic meaning for the individual. This leads to a fundamental shift in thinking. TIA is thus described as a move from "what is wrong with you" to "what happened to you"(Harris and Fallot, 2001).

The National Patient Safety Agency (NPSA) has a key role in the monitoring of incidents that put any patients at risk in any way. This applies across health service providers. In their guide Seven Steps to Patients Safety, the key factors to the creation of a culture of patient safety include the promotion of reporting, involving and communicating with patients and public and learning and sharing safety lessons. In addition, staff should report "near misses" or "no harm". NPSA recognises that there is a need for a greater awareness of the potential vulnerability of those experiencing mental health problems when there are inpatients. It is not the main focus of this paper but the NPSA note that this includes an acknowledgement that sexual disinhibition is a not uncommon symptom of serious mental illness. Therefore mental health professionals have to manage a number of different aspects of this problematic issue. However, it is vitally important to emphasise that the focus of interventions needs to be based on the potential victimisation of patients. 
NPSA guidance starts from the premise that patients' concerns must always be taken seriously. As noted above, one of the biggest barriers to the reporting of such incidents is the belief that victims hold that they will not be taken seriously and that this is, in part, due to their mental health problems. As well as emphasising that any allegations will be taken seriously and crimes investigated, the guidance highlights that patients should have access to appropriate sexual health advice and support services. The physical layout of the wards should also be considered. There have been moves to single sex wards more generally within health services. This appears to be of particular importance in this area. The Department of Health issued guidance recommending single sex sleeping, toilet and bath accommodation in 2000. In 2004/5, 99\% of institutions had completed these changes. The NPSA also notes that units that are in areas of high demand should resist the pressure to admit patients of the opposite sex to single sex wards in times of acute bed shortages. The wider pressure on mental health services makes this an increasingly common problem that mental health service providers have to face. The Royal College of Psychiatrists (RCP) has issued general guidance Do the right thing: how to judge a good ward on establishing a safe, secure and therapeutic ward (RCP, 2011). The RCP has produced a checklist of ten areas that includes: a proportionate and respectful approach to risk and safety and a physical environment that is fit for purpose. The guidance does not explicitly discuss sexual violence but emphasizes the importance of ensuring the physical safety of patients. It also highlights that separate toilets and sleeping accommodation for men and women is an important standard and a government policy. In themselves, these will not prevent incidents of sexual violence. However, they are all important in creating a culture that recognizes the potential increased vulnerability of inpatient, takes step to prevent incidents and provides appropriate support to those reporting any incidents of sexual violence.

National Reporting and Learning System (NRLS) exists to collate all reports of incidents. There is a general under reporting of incidents. The categorization of incidents includes patients accidents- for example slips and falls, disruptive and aggressive behaviour, self harm and absconding. In 2016, incidents categorized under these heading accounted for $84 \%$ of all reports. It should be noted that there is not a specific category of sexual assault or violence either as a victim or perpetrator. The NRSL carried out an analysis of a sample of 200 incidents of disruptive or aggressive behaviour. Five incidents in this sample concerned sexual safety. As a result, a further search of the whole data set of incidents of disruptive and aggressive behaviour was undertaken. There were 122 reported incidents where concerns were the result of sexual behaviour - 19 rapes, 20 cases of consensual sex, 13 cases of exposure, 18 
cases of sexual advances, 26 touching and 26 other. The report notes that there was significant variation amongst trusts in how they approach and report these incidents. When an incident is reported, the organization has to assess the degree of harm that the impact has had on the patient. In 114 of these cases, "no harm"was reported. This is a striking conclusion. The impact of such incidents has to include the potential traumatic impact - physical and psychological. These incidents may well be examples of revictimisation. In the cases of rape, 8 attacks were perpetrated by patients and 11 by staff. In addition, the 20 cases that are described as being consensual sex raise a number of important questions about the nature of consent in such circumstances. They also beg the question why consensual sex would be described as disruptive or aggressive

Developing appropriate, timely and sensitive responses to sexual violence is a key task for mental health professionals. Since 2003, it has been DH policy that all adult users of mental health services should be asked about their experiences of violence and abuse as part of mental health assessments - this was styled Routine Enquiry (RE). By 2006, it was clear that RE was not standard practice across mental health settings. Scott and McLeish (2008) carried out a case study analysis of trusts where RE was implemented. They found that it was a policy that required champions and key support from managers who ensured that it was team meeting agendas and so on to be successfully implemented. The main barrier to RE was staff resistance. Staff were concerned about the possible impact of asking such questions, not all were comfortable with discussing such issues. It was also felt that RE would only be justified if services were able to offer effective and better support to patients in these circumstances Survivors felt that their experiences of abuse were not seen as central or relevant to their mental health. In fact, this group indicated that they would have welcomed questions about these issues. The interviews that survivors had often had contact with a range of services who had missed opportunities to ask these questions. A culture of secrecy and shame is associated with abuse - a culture which abusers exploit - which makes victims vulnerable to revictimisation. Mental health professionals can help to tackle this culture by asking appropriate questions and as importantly following up with good services.

\section{Methodology}


The aim of this research was to investigate the extent of serious sexual violence on mental health units. The following Freedom of Information Act (FOI) request email was sent to 45 Police Forces across the UK.

"I am currently conducting a research study exploring serious sexual offences committed against individuals who were inpatients in psychiatric units at the time of the offence. Under the Freedom of Information Act 2000, I am writing to request anonymous information about recorded rape (Section 1 of the Sexual Offences Act 2003) and sexual assault by penetration (Section 2 of the Sexual Offences Act 2003) offences that have been reported and recorded by your constabulary between 1st January 2011 and 31st December 2015 involving a victim who was an inpatient in a psychiatric unit at the time of the offence - this group would include patients who were on section 17 leave from the hospital"

A data collection form was provided alongside the FOI request.

Following the responses from police forces, a similar FOI request was sent to MH trusts in England. A decision was taken to request information from Trusts in England and Wales was taken as no information had been provided by police forces in Scotland and Northern Ireland in response to the initial FOI inquiry. Incidents of sexual violence require responses from all agencies. These requests are part of a process of gaining a more detailed and or nuanced understanding of these complex issues.

Police Responses to FOI requests

\begin{tabular}{|l|c|}
\hline Total number of FOI requests & 45 \\
\hline Forces providing information & 23 \\
\hline Forces unable to assist & 22 \\
\hline
\end{tabular}

The forces that were unable to provide information cited the amount of time and the cost of retrieving the data. Local mental health unit addresses were not recorded in such a way as to make the information easily retrievable from databases. This would mean that each reported offence would have to be reviewed to check whether it took place on a mental health unit. An organisation can refuse an FOI request if it will cost more than $£ 450$ to retrieve the data. This was the response from 22 forces. There was considerable variation in the data provided by 
the 23 forces that provided some data. This alongside the geographical and demographic differences in the areas covered by the forces makes meaningful comparisons impossible on the basis of the information provided. For example, 10 forces provided on recorded s 1 and s 2 offences against inpatients. Even within this small sample, some forces gave aggregate data whilst others provided more detailed information such as the place where the reported offence took place.

As part of the FOI, forces were asked to complete an information sheet about the offences. These were often missing or incomplete. The information that the study was able to gather about the offences and the relationship between perpetrators and victims is provided in the following table

\begin{tabular}{|l|l|}
\hline Information from Police FOIs & \\
\hline Victims & 32 \\
\hline Female & 20 \\
\hline Male & 12 \\
\hline & 27 \\
\hline Perpetrators & 1 \\
\hline Female & 26 \\
\hline Male & \\
\hline & \\
\hline Relationship to victim & 10 \\
\hline Staff & 10 \\
\hline Fellow Patient & \\
\hline & 10 \\
\hline Place where the assault took place & \\
\hline Patient's room & 7 \\
\hline Hospital grounds & \\
\hline
\end{tabular}


When individual incident sheets were completed the information was incomplete. It was therefore difficult to reach strong conclusions about patterns of offending. However, the information provided indicated that women were at increased risk but both men and women reported assaults. From the limited information available from the police data, there were twenty female and twelve men victims. Information about the perpetrator was not always available. In cases where it was recorded, there were 26 males (two of the cases recoded involved two male perpetrators) and one female. The two most common group of perpetrators were fellow patients (10) or nursing or staff members (10). The offences were recorded as taking place in the patient's room (10), hospital grounds (7) or communal areas (6).

Mental health Trust responses

\begin{tabular}{|l|l|}
\hline Total number of FOI requests & 38 \\
\hline Trusts that provided a nil return & 13 \\
\hline Trusts that provided some information & 12 \\
\hline $\begin{array}{l}\text { Trusts that did not collect the data re- } \\
\text { quested }\end{array}$ & 12 \\
\hline Trusts that turned down the FOI & 1 \\
\hline
\end{tabular}

As with the FOI requests to the Police, there were signification variations in the reporting and collation of the data. A nil return by a MH Trust indicates that there were no such reported offences in the period covered by the FOI request. Six of the twelve trusts that did not collect the data advised the authors to contact the police for the information. The MH Trust that turned 
down the FOI did so on the grounds that complying with it might breach patient confidentiality. In these cases, there were four female and four male victims. No further information was provided about the location of perpetrators or their relationship to the victim.

\section{Conclusion}

The results of this research are consistent with the studies outlined in the introduction. Sexual assaults and rapes do occur in mental health inpatient units. The recording of incidents of sexual violence by MH trusts and police forces varies considerably. The Office for National Statistics (ONS) confirmed (email correspondence with the authors) that there is no national dataset for sexual offences that are committed on mental health inpatient units. These findings draw on small numbers and incomplete data but they highlight the variation and inadequacy of current recording practices in relation to sexual offences committed against inpatients. The data collected by MH Trusts obscures sexual violence as these incidents are recorded in a much generalized category of disruptive and aggressive behaviour. This system of recording also means that it is impossible to gain a clear picture of the extent and nature of this problem. Without this, comprehensive services to prevent sexual violence but also support those subject to it whilst inpatients, cannot be developed. Ellison et al. (2015) argue that we know very little about the extent of victimisation, police responses or how a mental health diagnosis or history influences notions of credibility for witnesses. These findings highlight a 'gap' in the recording of information in relation to rape. It may indicate that complainants with a history of mental illness are less likely to have their allegation recorded as a crime (Ellison et al, 2015). The findings also suggest that there is the concerns about patient safety that expressed in general policy, in practice there was considerable variation in how trusts responded to our request for information and the data they had available to them. What is clear is that trust responses to these allegations consider the role of the patient's underlying condition both in relation to the allegation and the substance of the at allegation (NPSA, 2006). Some trusts were willing and able to provide some overall statistical data but some made it clear these were allegations not convictions.

Existing research then would indicate that adult inpatients, especially women, are at increased risk of sexual victimisation and are less likely to have their allegations recorded as a crime. When they do they experience much higher rates of attrition than other rape cases (El- 
lison et al., 2015). Despite this background knowledge, the recording practices of both the police and NHS trusts remain hugely variable and incomplete. It seems evident that for adults with mental health histories/diagnosis, incidents of rape and sexual assaults, as with the adult population more generally, is underreported. From the scant information available, adults are likely to be at risk from fellow patients and members of staff and hospitals may contribute to, rather than diminish further victimisation. Given this situation, it seems imperative that we have more regular and accurate reporting of rape and sexual assault and that trusts show a greater willingness to record allegations and collate more detailed information of the incident and their responses to it. We would recommend that police forces explore ways of recording mental health unit specifically when dealing with such offences so the collation and analysis of data can be completed. We would also recommend that trusts and the NPA record these incidents separately from others of disruptive or aggressive behaviour. 


\section{References}

Bengtsson-Tops A, Ehliasson K (2012). Victimization in individuals suffering from psychosis: a Swedish cross-sectional study. Journal of Psychiatric and Mental Health Nursing 19, 23-30

Bloom, S.L. and Farragher, B., 2010. Destroying sanctuary: The crisis in human service delivery systems. Oxford University Press.

Care Quality Commission (2014) Monitoring the Mental Health Act 2012/13https://www.cqc.org.uk/sites/default/files/documents/cqc_mentalhealth_2012_13_07_update.pdf accessed 15th February 2017

Ellison, L., Munro, V.E., Hohl, K. and Wallang, P., 2015. Challenging criminal justice? Psychosocial disability and rape victimization. Criminology \& Criminal Justice, 15(2), pp.225-244.

Filer, N. (2013, The Shock of the Fall. London Harper Collins

Foley, M. and Cummins, I., 2015. Reading the death of Mrs A: a serious case review. The Journal of Adult Protection, 17(5), pp.321-330.

Goffman, E., 1968. Asylums: Essays on the social situation of mental patients and other inmates. AldineTransaction.

Harris, M. \& Fallot, D. (2001). Trauma-informed inpatient services. New Directions for Mental Health Services, (89), 33-46

Hester M (2013). From Report to Court: Rape Cases and the Criminal Justice System in the North East. University of Bristol in association with Northern Rock Foundation: Bristol.

Howard L, Trevillion K, Khalifeh H, Woodall A, Agnew-Davies R, Feder G (2010). Domestic violence and severe psychiatric disorders: prevalence and interventions. Psychological Medicine 40, 881-893

Jones, J., Nolan, P., Bowers, L., Simpson, A., Whittington, R., Hackney, D. and Bhui, K., 2010.Psychiatric wards: places of safety? Journal of psychiatric and mental health nursing, 17(2), pp.124-130.

Khalifeh, H., Moran, P., Borschmann, R., Dean, K., Hart, C., Hogg, J.,Howard, L. M. (2015). Domestic and sexual violence against patients with severe mental illness. PsychologicalMedicine, 45(4), 875-886. 
MIND (2007) Another Assault https://www.mind.org.uk/media/273466/another-assault.pdf accessed 19th July 2017

Nyame S, Howard L, Feder G, Trevillion K (2013). A survey of mental health professionals' knowledge, attitudes and preparedness to respond to domestic violence. Journal of Mental Health 22, 536-543

Paterson, B., 2014, June. Mainstreaming trauma. In Psychological Trauma-Informed Care Conference, Stirling University, Stirling (Vol. 4).

Pettitt, B., Greenhead, S., Khalifeh, H., Drennan, V., Hart, T., Hogg, J., Borschmann, R., Mamo, E. and Moran, P., 2013. At risk, yet dismissed: the criminal victimisation of people with mental health problems. www.vicitmsupport.org.uk accessed 19th July 2017

Quirk, A. and Lelliott, P., 2001. What do we know about life on acute psychiatric wards in the UK?A review of the research evidence. Social Science \& Medicine, 53(12), pp.1565-1574.

Royal College of Psychiatrists Do the right thing: how to judge a good ward on establishing a safe, secure and therapeutic ward http://www.rcpsych.ac.uk/pdf/OP79 forweb.pdf accessed 15th Febrauary 2017

Scott, S Williams, J and McNaughton Nicholls, C (2015) Violence abuse and mental health in England http://natcen.ac.uk/media/1057987/REVA Brief-1 Population patterns_FINAL_071015.pdf

Spandler, Helen (1996), Who's Hurting Who? Young people, self-harm and suicide, Manchester: 42nd Street.

Stenhouse RC. (2013) 'Safe enough in here?': patients' expectations and experiences of feeling safe in an acute psychiatric inpatient ward. J Clin Nurs;22:3109-18.

Sweeney, A., Clement, S., Filson, B., and Kennedy, A., (2016). Trauma-informed mental healthcare in the UK: what is it and how can we further its development?. Mental Health Review Journal, 21(3), pp.174-192. 